\title{
Collaborative Development of a PLE for Language Learning
}

\author{
doi:10.3991/ijet.v5s1.1196 \\ D. Renzel ${ }^{1}$, C. Höbelt ${ }^{2}$, D. Dahrendorf ${ }^{2}$, M. Friedrich ${ }^{3}$, F. Mödritscher ${ }^{4}$, \\ K. Verbert ${ }^{5}$, S. Govaerts ${ }^{5}$, M. Palmér ${ }^{6}$ and E. Bogdanov ${ }^{7}$ \\ ${ }^{1}$ RWTH Aachen University, Aachen, Germany \\ ${ }^{2}$ imc information multimedia communication AG, Saarbrücken \\ ${ }^{3}$ Fraunhofer Institute for Applied Information Technology, St. Augustin \\ ${ }^{4}$ Vienna University of Economics and Business, Vienna, Austria \\ ${ }^{5}$ Katholieke Universiteit Leuven, Leuven, Belgium \\ ${ }^{6}$ Uppsala University, Uppsala, Sweden \\ ${ }^{7}$ École Polytechnique Fédérale de Lausanne (EPFL), Lausanne, Switzerland
}

\begin{abstract}
This paper provides a report on the experimental collaborative and distributed development of a prototypic Widget-based PLE. The development process is described and detailed taking into account the requirements of a language learning scenario. First results are presented, and developer experiences are discussed critically with a focus on the development process as well as problems with current Widget technologies and interoperability.
\end{abstract}

Index Terms-Collaborative Software Development, Technology Enhanced Learning, Personal Learning Environment, Widget Interoperability.

\section{INTRODUCTION}

Current en deavors in the domain of Technology Enhanced Learning (TEL) exhibit the need for increased openness and $r$ esponsiveness of cur rent learning en vironments. While older learning technology generations were often central and closed systems, often merely focusing on the management of learning processes, the next generation of Personal Learning Environments (PLEs) tackled by the ROLE project ${ }^{1}$ concent rates on a hi ghly di stributed approach d rawing on the com bination of est ablished o penstandard Web technologies in order to enable the learnerside integration of servi ces and tools from a pl ethora of heterogeneous sources into c ustomized l earning en vironments. One of t he major goals of R OLE is to deliver an appropriate techni cal infrastructure for the establishment of such resp onsive open 1 earning envi ronments. Another goal of the project is to est ablish a co mmunity of ope $n$ source a nd e xternal devel opers out side t he conso rtium contributing further tools and services based on this infrastructure. D uring the first pr oject devel oper m eeting, we thus agreed to work in parallel to the standard project plan towards a comm on goal, cal led the "C hristmas Project" with the following objectives in mind:

- Create a first de monstrator of ROLE to visualize the potential of the project

- Enable the consortium to better define needs and derive technical specifications
- Experiment $w$ ith prom ising co mbinations of Web technologies towards an integration infrastructure for PLEs

- Explore the feasibility of a collaborative and distributed de velopment process scalable to a large com munity of independent developers

While th e first two ob jectives ad dressed th e con sortium-internal collaboration, the las $t$ two clearly address a broader audience. Thus, this paper reports on the results of the Christm as Project with a focus on the collaborative distributed development process and a first integrated PLE prototype resulting from this process. It gi ves the reader an insight into the challenge $\mathrm{s}$ we faced during our work regarding the development process and the technologies we experimented with. After drawing the conclusion that current t echnology and devel opment processes are oft en still in sufficient for a sea mless in dependent development of in teroperating learn ing serv ices an d to ols, i t o utlines possible improvements.

The rest of $\mathrm{t}$ his doc ument is structured as follows. In Section II we describe the development process to give an insight of how our work was organized. In S ection III we present d etails on the req uirements el icited fo $r$ an in tegrated PLE ba sed up on a 1 anguage l earning scenari o. In Section IV we present the individual partner contributions in more detail. Section V presents our expe riences and a critical conclusion of o ur wo rk reg arding cu rrent issues regarding Widget technology. In Section VI we end with a short summary and give a short outlook to further work.

\section{DEVELOPMENT PROCESS}

Following the objective of est ablishing a comm unityoriented development process, we planned to explore such a process in a smaller scale within the consortium starting off with a co mmunity of $n$ ine part ners a cross Eu rope, from both industry and acade mia and with different degrees of $t$ echnical backgro und. Since heavy-weight processes would in practice not be feasible and accepted with a large-scale developer comm unity, we decided to keep the process as 1 ight-weight as possible, ho wever bo rrowing concepts from standard processes such as A gile Development [1][2], e.g. short itera tion cycles, shared code \& documentation, cont inuous integration, re gular devel oper

\footnotetext{
${ }^{1}$ http://www.role-project.eu/
} 
communication, etc. However, given by the spatial distribution of the community, concepts requiring phy sical attendance em phasized in Agi le approaches had t o be replaced by comm unication tec hnology in orde $r$ to avoid roundtrip u nrealistic in a larger scale comm unity. Furthermore, such a distributed approach re quires t echnical means for code and documentation sharing and an integration envi ronment with low en try barriers. Table I bri efly shows the rather light-weight abst ract schedul e we $p$ ursued during our experiments. It should be noted that this process can be i terated. Ho wever, t he sc hedule sh own here is likely to be $\mathrm{s}$ ubject to refinem ent or even re placement in next phases of the ROLE project.

In the following, we provide details on the first iteration conducted during the ROLE Christmas Project.

All of the participants dedicated themselves to contribute co mponents for an i ntegration fram ework, individual learning servi ces, ei ther im plemented or a s mockup to reach the comm on goal of deliv ering an integrated PLE prototype.

After the collection of all contributions intended by the partners, we s ketched the R OLE Christm as Project Big Picture (cf. Fi gure 1). Given by the het erogeneity of $t$ he partners' plans for contributions, we agreed on a common scenario serving as a concret e use case for a ROLE PLE prototype base d on a Widget approach. For that pur pose we chose a language learning scenario described in detail in Section III.

As a basi s for on going d ocumentation we deci ded to setup a document to be edited collaboratively by all partners, starting with the Big Picture, an elaborate description of the scenario and a tim e plan. Every pa rtner adde $\mathrm{d}$ a description of his contribution and how it would fit with the scenario. Thereby, we did not require a perfect match, but at least a high degree of relevance.

A ROLE XMPP Server was s etup for direct communication. A R OLE devel oper c hat room was confi gured to log all group conversations on the server side. Thus, everybody c ould easily keep t rack o n previous di scussions, which turned out to be a helpful feature. However, restrictive firewall $\mathrm{p}$ olicies en forced by $\mathrm{v}$ arious partn er in stitutions so metimes hindered the use of XM PP - a val uable experience for fut ure consi derations regard ing $i$ ts use i $n$ our software (cf. Section IV.E.)

TABLE I.

ABSTRACT PROCESS SCHEDULE

\begin{tabular}{|c|}
\hline Planning Phase (collaborative) \\
\hline 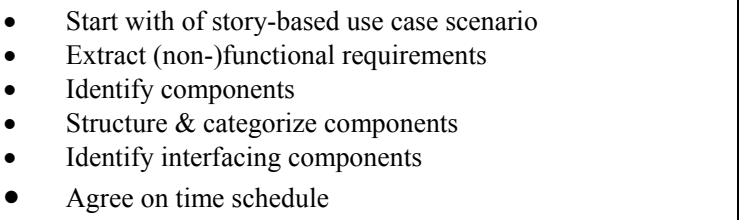 \\
\hline Development Phase (distributed \& independent) \\
\hline $\begin{array}{l}\text { - } \\
\text { - } \\
\text { - } \\
\text { - } \\
\text { - Come own development environment } \\
\text { - Continuously share code \& documentation } \\
\text { tion environment }\end{array}$ \\
\hline
\end{tabular}

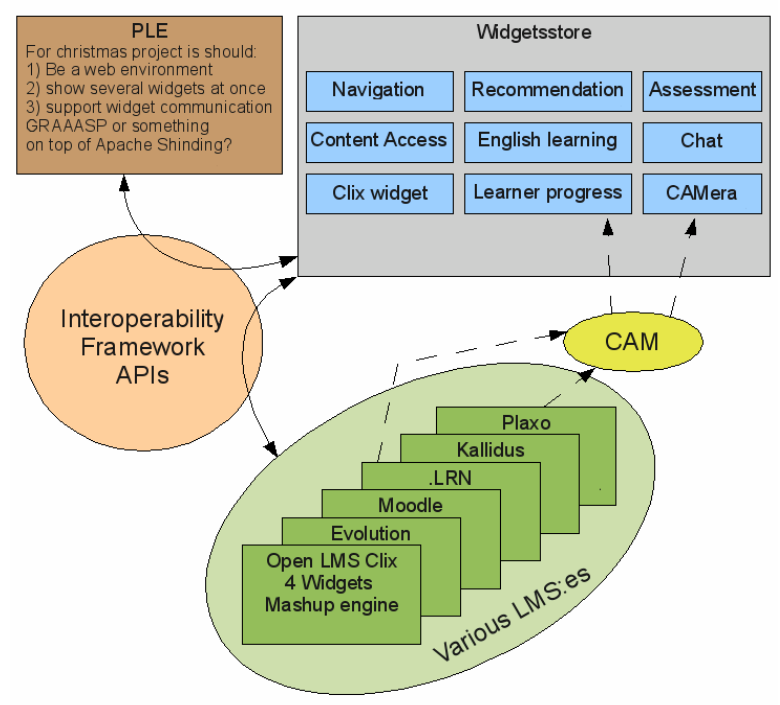

Figure 1. ROLE Christmas Project Big Picture

Furthermore, in order to maintain the source code of all partner con tributions, we ag reed o $\mathrm{n}$ u tilizing a $\mathrm{g}$ it-based [3] repository at github.com for reasons of wide visibility and acceptance in the open source developer co mmunity. Besides SCM fun ctionality, g ithub prov ides an issu e tracker, Wikis, rep ository statis tics, etc. All of these features were frequently used during the development phase.

With regard to a development environment for the individual part ner cont ributions, we defi ned t he fol lowing simple policy:

1. All partners setup development environments.

2. One partner $m$ aintains i ntegration envi ronment and regularly pulls from the ROLE github.

During a later physical meeting we discussed a few options and suggestions regarding the choice of technologies as a basi s for development and i ntegration environments. The following considerations were taken into account:

1. How to quickly move forward and s ucceed with Christmas Project on schedule.

2. Avoid using te chnologies that will hinder us or force us to start over completely later on.

3. Make as few decisions as $\mathrm{p}$ ossible at this point in time.

Since the prot otype should be Widget-based, we discussed a pre-selection of promising technologies for software com ponents such as W idget engi nes, cont ainers, stores and $\mathrm{r}$ epositories, $\mathrm{i}$ nter-Widget communication mechanisms, protocols, etc. and st arted our developments after a first decision for one configuration.

All co mponents of $t$ he so ftware we used a s t echnical foundation $\mathrm{f}$ or our wo $\mathrm{rk} \mathrm{p}$ romised $\mathrm{t} \mathrm{o}$ be $\mathrm{pl}$ atform and browser independent - unfortunately, this assumption was far from being fulfilled. It turned out that all regarded alternatives were still in an early experimental development stage. The consequence was an increased communication overhead am ong the part ners for $t$ he purpose of fi nding, agreeing on a nd cha nging to $\mathrm{m}$ ore acceptable solutions. Due to the fact that we could never rely upon the software components of our development and i ntegration environments, continuous integration was hardly possible, leading to the usual longer and error-prone final integration phase 
shortly before the prototype delivery deadline. The details of the technical proble ms we faced will be discussed in Section V. In th e $\mathrm{n}$ ext sectio $\mathrm{n}$ we present $\mathrm{d}$ etailed requirements for the ROLE Christmas Project.

\section{REQUIREMENTS}

In th is section we will $\mathrm{p}$ resent an o verview of the requirements el icited for $\mathrm{t}$ he $\mathrm{r}$ ealization of $\mathrm{t}$ he $\mathrm{C}$ hristmas Project. We start with the lan guage learning scenario we agreed upon as well as the underlying psycho-pedagogical model. We then continue with technical requirements for the realization of our prototype and considerations on how to fulfill them.

\section{A. Scenario}

In our language learning sce nario, the learner, Tim, is an employee at Travel Books that sells books and vi deos on travel dest inations. He w orks in the sal es departm ent and has to go to international fairs an d to speak with distributors, bookshops and other business partners. As most business com munication is in En glish, Tim needs t o improve his English skills, especially in Business English.

One part of his learning strategy is to read texts and to learn its vocabulary using his PLE. For that purpose he adds three widgets: a Language Resource Browser, a Vocabulary Trainer and a Translator widget. All of them are visible on one webpage.

In the Langua ge Resource Browser, Tim searches for a text and st arts reading it. Each t ime he misses a word he selects it and opens a context menu on it. The syste $m$ then proposes him to ei ther look it up in the Translator widget or send it to the Vocabulary Trainer widget (cf. Figure 2).

So he adds words that he con siders as important to the Vocabulary Trainer and others he only looks up.

After reading the text, he has gathered a list of words he considers important to be repeated in future using the Vocabulary Trainer widget.

In the next day s he cont inues reading new spaper art icles regularly and his V ocabulary Trainer widget obtains more and more words. In an analogous manner he uses the Language R esource B rowser wi dget t o wo rk wi th ot her media types such as audio or video.

One day, he i s l earning wi th hi s Voca bulary Trai ner, memorizing the words o $\mathrm{n}$ the list and testing whether he knows them suffi ciently well. He recogni zes that he has problems to remember a certain word because he does not know the context anymore where it originally appeared.

Fortunately the Vocabulary $\mathrm{Tr}$ ainer always stores the link to the original text. So Tim clicks on the word and the original t ext appears i $\mathrm{n}$ the Language R esource B rowser widget and shows the sentence where the word was taken from.

Reading the sentence and thinking of the context facilitates him to memorize the vocabul ary. Furthermore he improves his language proficiency by knowing situations where he can use the word.

The scenari o c an be e xtended by a group of 1 earners, e.g. st udents t hat part icipate $\mathrm{i} n$ an En glish Lan guage course. The instructor sends them a list of newspaper a rticles that are a vailable online. The students are asked to read and anal yse the $m$ and to learn the vocabulary. As they come from the same background (high school Eng lish level), they decide to jointly create a vocabulary list

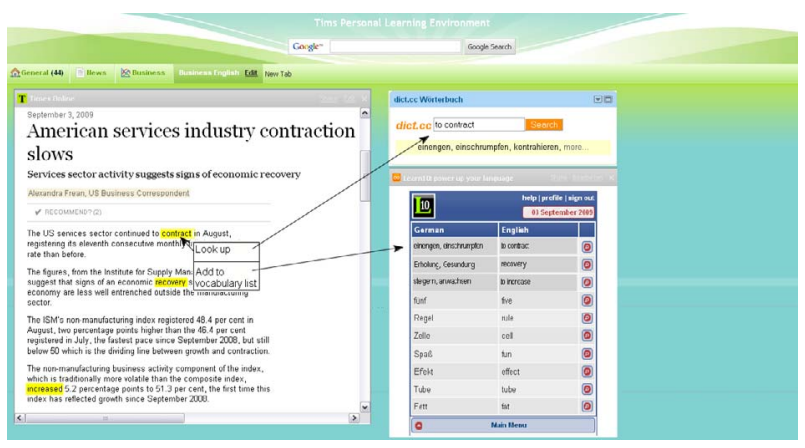

Figure 2. PLE with three Learning Widgets

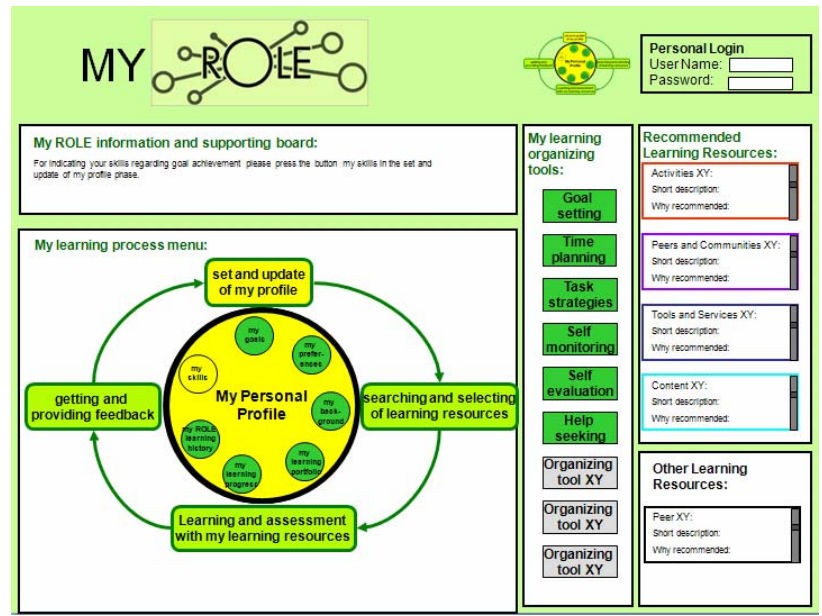

Figure 3. Learner Navigation Tool Mockup

using the Vocabulary Trainer widget. Whenever a student finds an unknown word in the newspaper article, he adds it to th e j oint v ocabulary list. Th e Vo cabulary Train er widget will al so d isplay word s add ed b y other stud ents who have the same level in the En glish language. Words that have been added $m$ ore often are sort ed hi gher. Each learner in dividually tra ins the lis $t$ of words an $d$ th e vo cabulary widget keeps track of each student's individual vocabulary kn owledge. The group's averag e knowl edge (number of words learned) is displayed in the widget, together with the current student's knowledge. Nevertheless it should be possible to deactivate the group functionality if the learner only wants to learn for herself.

\section{B. Psycho Pedagogical Model}

In th is section we sh ortly p resent the ROL E p sychopedagogical model [4]. The central element of this model is a cy clic learning pr ocess model consi sting of seve ral learning $\mathrm{p}$ hases related to learning activ ities. Fo llowing the connections between lear ning ph ases, activ ities an $d$ tools, a seque nce of 1 earning tools can be deri ved. Fu rthermore, two different kinds of 1 earning tools are i dentified, fi rst "no rmal" learning t ools con veying d omain knowledge, and second, meta-learning tools used for selfregulating the own learning process.

A na vigation $t$ ool $g$ uiding the 1 earner $t$ hrough a sel $\mathrm{f}$ regulated learn ing pro cess by reco mmending activ ities and tools was proposed. The partners created an i nteractive mock-up of such a na vigation tool (cf. Figure 3 ) and presented it in the context of the scenario from the previous section. 
The contribution of education professionals had a great impact on the technical developments and showed us once more that technical and co nceptual wo rk s hould be conducted hand-in-hand.

\section{Technical Requirements}

For the technical realization of a first ROLE PLE prototype, we decided to follow an approach of intercommunicating W idgets. The expect ations from choosing such an approach were a rela tively loose cou pling bet ween individual wi dgets cont ributed from di fferent part ners. It should be not ed that we al so experimented with such an approach, because i n f uture pr oject st ages, devel opers outside the co nsortium shoul d be e nabled to w ork com pletely independent from other developers.

First, a technical infrastructure was needed as a basis for our prototype. Starting from the Big Picture, we identified requirements for the following components of such an infrastructure:

1. Widget Container/Engine

2. Widget Store/Repository

3. Widget Interoperability Mechanisms

4. Widget User Interface

As pr omising $\mathrm{W}$ idget $\mathrm{C}$ ontainer/Engines, we consi dered ex periments with Apache Shindig [5], the reference implementation of an OpenSocial [6] container currently under i ncubation at Apache. OpenSocial defines a co mmon API fo r so cial ap plications acro ss multiple web sites and also includes a s pecification for widgets - gadgets in OpenSocial terminology. As further alternative we co nsidered Soci alSite [7], based on Gl assfish and A pache Shindig with the ab ility to ru $\mathrm{n}$ OpenSocial g adgets an $\mathrm{d}$ have them backed by the same social graph. Furthermore, Apache Wookie [8] was tak en into consideration as a so lution for adding W3C Widgets [9] as well as OpenSocial and Google (Wave) Gadgets to web applications.

1) As solutions for a Widget store/repository, we considered the following four solutions:

- No store/repository, fixed list.

- Wookie

- Google Gadget directory

- ROLE widget store (remains to be built)

A fun damental req uirement to Widget in teroperability was t he supp ort of $i$ nter-widget com munication. There should be no major co nfiguration needed on the si de of end users asse mbling wi dgets. Furt hermore, it shoul d be easy to build containers for the chosen widget technology. Integration into existing systems, like LMS should be possible. Preferably, standard co ntainers should al so support these technologies. The following technologies were taken into closer consideration as potential candidates:

- Gadget pubsub [6]

- OpenAjax Alliance hub2.0 pubsub [10]

- Open Application (draft) [11]

- XMPP [12],[13] XEP-060 Publish/Subscribe [14]

- HTML5 DnD[16]

2) Regarding a Widget user interface a couple of diffe rent approaches were considered, e.g. portal pages suc $h$ as iGoogle, Wiki or LMS approaches, etc. However, we decided $\mathrm{t} o$ kee $\mathrm{p} t$ hings si mple fi rst usi ng a fixed $\mathrm{H}$ TML page. A $\mathrm{n}$ addi tional sol ution experi mented with was the integration in Graaasp (http://graaasp.epfl.ch), a W eb 2.0 contextual aggregator of peop le, spaces, assets and tools, in order to be able to make use of its built-in mechanisms for sharing, commenting, and recommendation.

For the first experiments, we decided to use a configuration of SocialSite as a container, no widget store, simple HTML page or Graaasp as us er interface and OpenApplication based on Gadget pubsub for inter-widget communication. Furt hermore, $\mathrm{t}$ he pr eviously desc ribed $\mathrm{R}$ OLE XMPP Server was used for e xperiments on rem ote interwidget co mmunication. Fo $r$ th is first $p$ rototype, th e $p$ rimary focus was thus put on the development of communicating widgets.

\section{PARTNER CONTRIBUTIONS}

In this secti on we wi 11 present the contributions of al 1 partners for the Christmas Project prototype. The first two contributions are $\mathrm{t}$ argeting at an $\mathrm{i}$ ntegration $\mathrm{f}$ ramework focus on the communication between widgets. All remaining contributions consist of $d$ ifferent types of wi dgets either especially for the langua ge learning scenario or with rather general functionality.

\section{A. Inter-Widget Communication}

Uppsala U niversity introduced t he Ope $\mathrm{n}$ Appl ication Event API to provide a generic solution to the requirement of inter-widget communication in the scena rio. The $\mathrm{m}$ ost important aspect of the solution is that wi dgets need not be "hard-wired" against ea ch other. Instead, they communicate using well-known data expressions, with the intention that widge ts will underst and the parts that are important to them.

The basic principle behind the event API is that all widgets are $\mathrm{n}$ otified of all ev ents. No sp ecific su bscription step is necessary. All widgets are given the opportunity to react to any event, which the y may choose to do depending on event type, message type, message content, etc.

\section{1) Events types}

The event typ es include: state, load, modify, save, select, unselect, startDrag and stopDrag. A state event is different fr om $\mathrm{t}$ he ot hers in $\mathrm{t}$ hat it has no rel evant resource, and therefore no resource is sent along. Instead, a state event indicates a change of state in the wid get that sent it.

\section{2) Event Structure}

Notifications of events are se nt out as messages. The message consists of the relevant resource, if any, depending on the event. The message is wrapped in an en velope containing further event information.

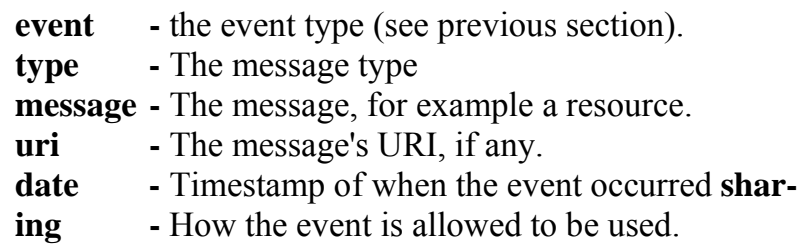

At the m oment, the event API consists of a Gadgets PubSub channel, in which the messages are published and thereafter sent out to all widgets.

An Open Application co mpliant wid get sub scribes to the PubSub channel when the widget is loaded. The shar- 
ing prop erty is in tended to sp ecify how the data may be used: on the same page, only on the user's machine, by a service under $t$ he user's co ntrol, by part icipants with access to the same widget instances, or that it may be transmitted to $\mathrm{v}$ arious serv ices. Each lev el includes all the privileges of the previous levels.

3) Message Types

A number of message types are defined. These include: namespaced-properties, JDIL, JSON, URL, HTML, XML and MIME content. Of thes e, nam espaced-properties is intended for simple RDF-like metadata with direct properties, and MIME content for unparsed text or binary data.

\section{B. Web 2.0 Platform for Collaborative Organization of Information and Tools}

The EPFL team developed a Web 2.0 platform, namely Graaasp, to helps users to collaboratively organize information and tools toward a given goal or activity. Tools in Graaasp are im plemented a s widgets. In addition to the standard add, remove, browse, group and share operations, Graaasp also supports taggi ng, rating a nd commenting. The widgets are imported/bought from a wi dget store and linked to the given Graaasp s pace dedicated to a learning activity.

Once the activity is co nfigured the user can switch the view to play with instances of selected widgets. The widget instances are rendere $d$ in a wi dget container managed by a $\mathrm{W}$ ookie engi ne. Any wi dgets following the W3C widget specifications can be instantiated into Graaasp.

Based on both the created activity structure and the user ratings, a recomm ender sy stem that woul d cont extually recommend $\mathrm{w}$ idgets to use rs is bei ng de veloped. Similarly, Trust and Reputation algorithms for widgets are also considered.

\section{Monitoring of User Behaviour}

To provide recommendation and self-evaluation mechanisms Fraunho fer FIT devel oped a C AM [17] wi dget to unobtrusively m onitor user behavi or. Other wi dgets, like the voca bulary $t$ rainer wi dget (cf. next sec tion), $t$ rigger events on different user act ions w hich are then br oadcasted $t \mathrm{o}$ ot her wi dgets using $\mathrm{O}$ pen Application. The CAM schema provides a standardized data format to store user activ ities an $\mathrm{d}$ thu $\mathrm{s}$ fo sters wid get in teroperability since every widget could access these data.

Since the CAM widget is a simple subscriber widget, it listens to every event published from any other widget and collects the $\mathrm{m}$. The collected ev ents are then transferre $\mathrm{d}$ into the CAM schema and afterwards stored in a database. As all control should be with the user, she can decide between different storage modes. For $\mathrm{t}$ he Christmas project we therefore specified three different storage modes which can be selected by the user (cf. Figure 5).

If the u ser decides to sto re her activ ities re motely, the CAM wid get transfers the data to a cen tral CAM repository, where all events of every user are stored. After creating a CAM instance, the CAM widget calls a Web service [18] passing the CAM information which is then stored in a database. The local storage mode uses the Gears plug-in [19] to st ore CAM i nformation in a l ocal database. The Gears pl ug-in is avai lable for $\mathrm{m}$ any $\mathrm{pl}$ atforms and supports all common browsers. It provides a S QLite [20] interface to easily create a database and store information

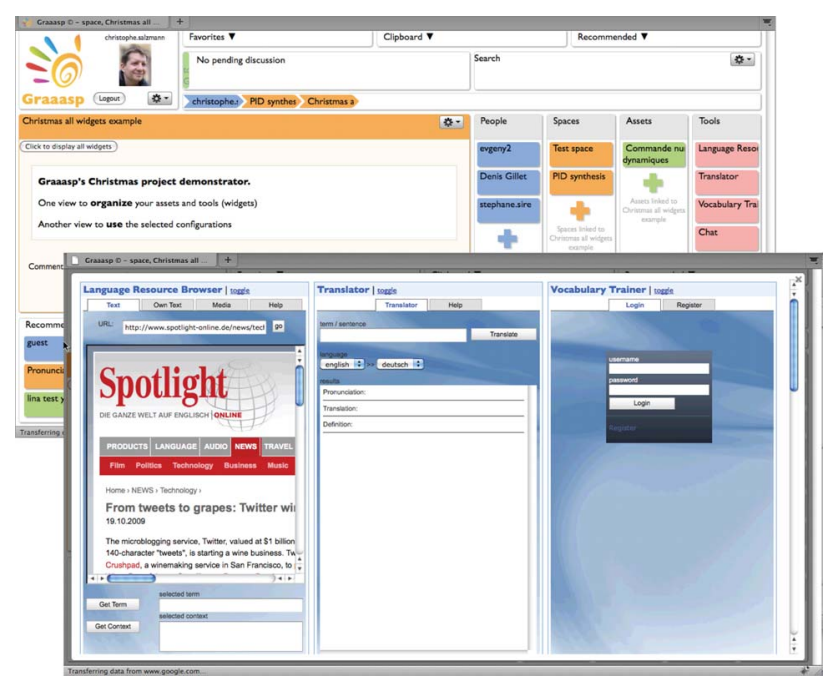

Figure 4. Graaasp views to organize the activity (on the back) and to play the instantiated widgets (on the front)

CAM $\mid$ settings toggle
Select CAM storage Mode:
$\bigcirc$ remote
$\odot$ local
$\bigcirc$ off
last 10 entries...

Figure 5. The CAM widget with three different storage modes

inside of $t$ he local browser profi le. An altern ative ap proach to Gears is using HTML5 [16]. Since the specification of HTM L5 is n ot fi nished y et and i s currently not supported by every browser we have chosen Gears. If the user does $n$ ot want her usa ge behavi or st ored, s he can choose the storage mode off.

To ge nerate $r$ ecommendations based o n CAM, $t$ hese can either be based on the user's own previous behavior or the usage history of others can be taken i nto account as well. The di fferent storage modes have effect on the generation of recommendations. In the remote mode the user can get reco mmendations, but al so al lows the sys tem to use his information to generate recommendations to other users. The local storage mode only allows retrieving recommendations, and disallows the system to use her information to generate recommendations to other users. If the user does not want her usage behavior to be monitored she can neither get recommendation nor support the system to generate recommendations to other users.

As we ha d no Vocabulary Trainer CAM data for the Christmas proj ect, we $t$ ransferred som e PLE $M$ onitoring data [21] into the CAM schema and provi ded a separat e Web service which offers methods to generate recommendation and self evaluation statistics.

\section{Visualization of Monitored Activity}

KU Le uven d eveloped a da shboard that enabl es st udents and teachers to $\mathrm{m}$ onitor learning activities. In the dashboard students, can monitor the progress they made 


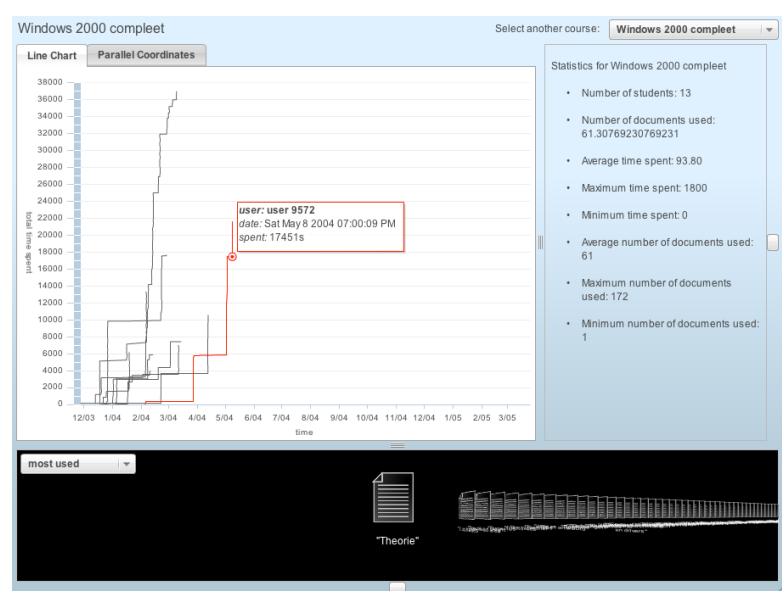

Figure 6. Dashboard with line chart

on a certain co urse or task and compare themselves with other students working on the same task.

An important feature of the dashboard is learning material recommendation. Based on the learning material other students have used, who have progressed further, we can recommend interesting learning material to the student. A student can compare his activities with other students and a teacher can get a general overview of what is going on in the course, see if it meets expectations and detect potential problems.

Figure 9 illustrates the first version of the user interface for our application. The st udent can sel ect the course and will be presented with 2 different charts, a course analytics overview and document recomm endations. Every line in the chart in figure 1 is a st udent. The chart shows when the st udent worke $\mathrm{d}$ (h orizontal axis) and how $\mathrm{l}$ ong h e worked on the course (vertical axis). The red line shows a selected student, we see that the student was very late in finishing the course and that he spent a lot of

work in a s mall number of sessions. This view enables a student to compare his progress wi th that of hi s fellow students. Another vi sualization uses pa rallel coordi nates [22]. It shows a set of metrics on parallel axes. A st udent is represented as a polyline with the vertices on every axis. The metrics are: the average and to tal time spent on the course, the n umber of docu ments used and the average time of the day that a student works. By visualizing these metrics next to each other one can grasp another view on the course activity and discover trends.

The wi dget is devel oped in Ad obe Fl ex. To m onitor user activities, we use CAM [17]. In order to test the tools, we used course data provided by U\&I Learning [21]. Together with our partners at FIT, we experimented with the data $t$ o propose possi ble $m$ etrics and c ollaborated o $\mathrm{n}$ a Web service providing methods to retrieve and cal culate: a list of all courses, a list of reco mmended documents for a course, gene ral statistics for a course, st atistics of a st udent of a course and the student's attention metadata for a course. The wi dget can be easi ly deployed on t op of another Web service that uses different attention metadata to provide the same statistics.

In a teacher modus, the stude nt names may need to be anonymized. Privacy is an important issue when monitoring this kind of data. For the Christmas project, we want to sim ply anonym ize $t$ he nam es of $t$ he st udents i $n t$ he teacher view. This is not im plemented ye $t$, because the data from U\&I was already anonymized.

Another design idea, n ot y et implemented, is a graph based com munity vi sualization widget. T his t ool coul $d$ allow students to find fellow students how have the same language proficiency as them to chat or col laborate with. The widget would communicate with the chat module of RWTH to provide chat func tionality. W e are cu rrently implementing this widget.

\section{E. XMPP Chat Widget}

The contribution of RWTH Aachen University is a Chat Widget providing a si mple Instant Messaging (IM) client (cf. Figure 7) based on the XMPP [12][13] protocol. The widget offers interface elements for 1-on-1 conversations, the management of buddy lists and user presence information. In $\mathrm{i}$ ts defaul t confi guration, the wi dget connect s to the ROLE XMPP Server. However, connections to arbitrary XMPP Servers are pos sible. Reg arding th e ROLE Christmas Project's language learning scenario, the XMPP Chat $\mathrm{W}$ idget cont ributes to s ynchronous co mmunication between learners integrated in a PLE. T he widget is as a rich source for communication events, e.g. updated presence i nformation, i ncoming/outgoing $m$ essage, etc. The integration of event publishing in to the Open Application approach to be captured by CAM is planned for the near future.

Besides the added value of XMPP chats between learners and basic c ommunication statistics, these two widgets demonstrate how i nter-widget co mmunication co uld be realized between rem ote wid gets (via sendi ng m essages over XMPP) and between local widgets (e.g. vi a Gadget pubsub). There already exis t specifications on XMPP EXtension Prot ocols (XEPs) f or Publ ish/Subscribe [14] or Personal Eventing Protocol [15] mechanisms, which will be conside red for a seam less re mote/local inter-widget communication for future developments.

\section{F. Language Learning Widgets}

In order $t$ of ulfill the requi rements of $t$ he 1 anguage learning scenario, imc AG developed the English learning widgets: The Langua ge $\mathrm{R}$ esource $\mathrm{B}$ rowser wi dget, $\mathrm{t}$ he Vocabulary Trainer widget a nd t he Trans lator wi dget. These widgets demonstrate a reasonable use of the Interwidget communication by se nding a nd receiving ter $\mathrm{m}$ items described by a term and its context and source.

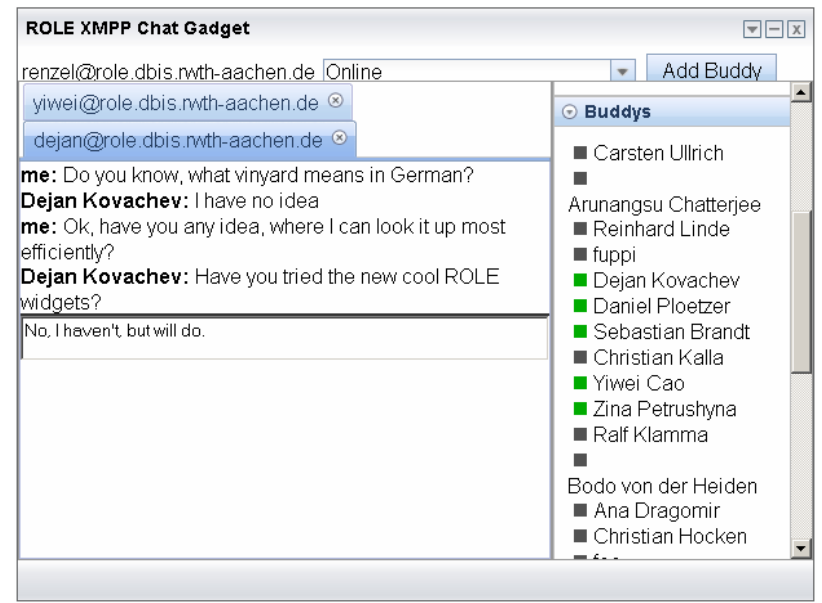

Figure 7. ROLE XMPP Chat Widget 


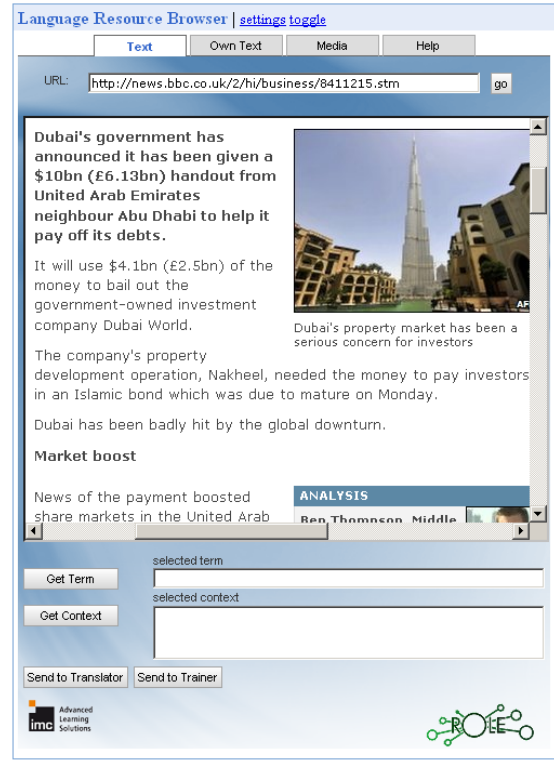

Figure 8. Language Resource Browser Widget

\section{1) Language Resource Browser Widget}

The Language Resource Browser widget (cf. Fi gure 8) allows u ser to co nsume media an $\mathrm{d}$ sen $\mathrm{d}$ ter $\mathrm{m}$ ite $\mathrm{ms}$ to other wi dgets processi ng the i nformation. Exam ples of such widgets are the Translator Widget where the ter $\mathrm{m}$ will b e tran slated or the Vo cabulary Train er wh ere th e user can add this term to a vocabulary list. At the moment the widget offers three different tabs. The "Text" tab works like a web $b$ rowser. It di splays a page $t$ o a gi ven URL in an iframe where the user can select the ter $\mathrm{m}$ and context. The source of such a term item will be the URL from the page.

In the second tab "Own Text" the user can add her own text taken from an online or offline resource. The third tab provides su pport t o browse for different media such as video and audio. While watching or listening to the media, the user can enter a term in a field. The s ource of suc $\mathrm{h}$ a term item will be the URL from the media and the context will be defined as "Media Context".

\section{2) Translator Widget}

The Translator widget allows a user to translate terms or sentences. It translates either a term which was entered from the user or a received term item. We combined different Web se rvices (i .e. Wikipedia, G oogle Di ctionary, DICT.ORG, Google Translate) for the translation process

At the moment o nly En glish to Germ an is su pported, but the language pool could be extended to all languages supported by the services above.

\section{3) Vocabulary Trainer widget}

The Vocabulary Trainer (cf. Figure 9) widget is implementing a sli ghtly modified Lei tner sy stem [23]. A vocabulary list consists of five different buckets. If an item is added is will be put in the first bucket. If the $u$ ser is training a $l$ ist and knows the right translation the item will be moved to the next bucket and else it will be moved to the previous bucket.

The inform ation is stored on a central server and accessed using REST Web services. Each user has a unique login and authentication is done by basic access authentication over REST. For translation the same Web services

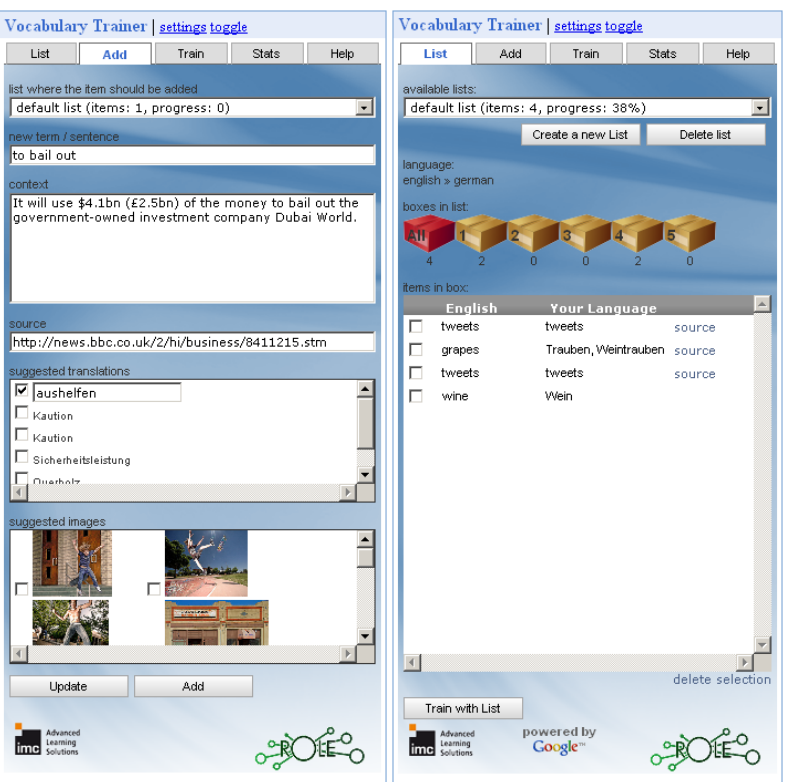

Figure 9. Vocabulary Trainer Widget

are used as in Translator widget, and Flickr is used to suggest pictures for term $\mathrm{s}$. Vocabulary ite $\mathrm{ms}$ are stored in a list which can be managed by the user.

The widget has four functionalities represented by four tabs: "Add", "List", "Train" and "Stats".

The "Ad d" tab allo ws u sers to manually in sert a $\mathrm{n}$ ew term/sentence, the context of that term and it s source. In combination wi th $t$ he Lang uage $\mathrm{R}$ esource $\mathrm{B}$ rowser $\mathrm{t}$ he sent ter $m$ ite $m$ appears auto matically in the Vocabulary Trainer widget.

The "List" tab provides an overview of the stored lists and vocabulary items. The us er can create/delete lists and inspect the content of the different buckets.

The "Train" tab gives the learner the possibility to practice her st ored vocab ulary. Aft er ch oosing a buc ket that she wants to train a ter $m$ from th is bucket and its co ntext will be displayed. The user can $g$ et help by viewing the source of that item or vi ewing the image to th at item (if there exists one). The fourth tab "Stats" shows statistics of the training. It displays a global score and a score for each list.

\section{G. Federated Search \& Language Processing}

In traditional educational s cenarios, teachers typica lly provide appropriate learning materials and gi ve feedback on st udent essay s. Vi enna Uni versity of Economics \& Business cont ributed $t$ wo wi dgets whi ch a re useful for these purposes and indicate their application for language learning.

ObjectSpot, a widget for federated search of academic papers in $\mathrm{d}$ ifferent digital lib raries, $\mathrm{h}$ as its origins in the iCamp project (http://www.icamp.eu). This search client allows plugging in different di gital repositories, whereby the docum ents are ret rieved via the Sim ple Query Interface (SQI) [24] standard. The central core of this widget is the ranking algorithm which has been developed over several itera tions an $\mathrm{dm}$ ixes in the search resu lts fro $\mathrm{m}$ the repositories on the fly [25]. In practice, this widget is useful for both learners and teachers to retrieve the most appropriate litera ture for a sp ecific knowledge d omain. By default, $\mathrm{t}$ he $\mathrm{m}$ ost im portant di gital 1 ibraries cont aining 
academic papers, e.g. ACM, IEEE, Google Scholar, CiteSeer, EBSCO, etc., are included.

The screenshot shows the re sults fo $r$ th e q uery ter $\mathrm{m}$ 'open responsive learning en vironments'. At the top, the search term and the state of the repositories are displayed. At the bottom, the user can navigate through the pages. On the left hand side, a user can 'lock' appropriate results, thus giving explicit relevance feedback and recommendations for others. This mechanism can al so be used to export search re sults through a feed-based API if another tool is attached to ObjectSpot. Pressing the option button, a user can co nfigure SQI-enabled di gital reposi tories for her search client, visualize th e location of the repositories on a map, get statistics on the quality of the repositories, export the results as RSS-feed, get recomme ndations for a query term, or plug another tool to ObjectSpot.

Conceptalyzer comprises a language processing widget which builds upon a LS A-based Web services developed within the LTfLL project (http://www.ltfll-project.org).

This wi dget anal yzes onl ine resources (e.g . Wikipedia articles or RSS feeds) in terms of the concepts behind the text and visualizes them according to their relevance. Application areas of this widget comprise learner positioning, monitoring on e's conceptual development. It can be al so helpful for teachers in pre paring learning materials or grading students [26].

The screenshot shows the re sult of the an alysis of a Wikipedia article, whereby th e rel evant terms are vi sualized in the form of a 'concept cloud'. The size of a t erm indicates its relevance for the article while the color links to the text corpora used to train the LSA function.

\section{Discussion of Widget TeChNOLOGIES}

During the de velopment pro cess of the ROLE Christmas Prototype PLE, we collect ed a set of valuable however negative experiences to be s hared with other developers working with the tec hnologies we atte mpted to combine. These experiences will be discussed in this section.

One of the most surprising experiences from the developer perspective was the immature state of many widget technologies, especially with regard to inter-widget communication, one of ou $\mathrm{r} m$ ain requi rements for $\mathrm{t}$ he 1 anguage learning PLE. Duri ng the development process we tested the fo llowing th ree OpenSocial co mpliant Gadget development environments:

1. Apache Shindig

2. SocialSite (based on Shindig within Glassfish)

3. OSDE (Eclipse Plugin with integrated Shindig)

With all of the above sy stems we encou ntered at least one of the following problems:

- Lack of Forward Incompatibility

- Client Browser Dependence

- Server Platform Dependence

- Inaccessible Bugs in Generated Code

- Incompatibility with External Libraries

- Lack of Developer Support

The first problem was relate $d$ to the in stallation of a gadget co ntainer, in p articular with So cialSite. F irst, the current $\mathrm{S}$ ocialSite di stribution is restricted to specific, already outdated versions of Glassfish and Shindig, and

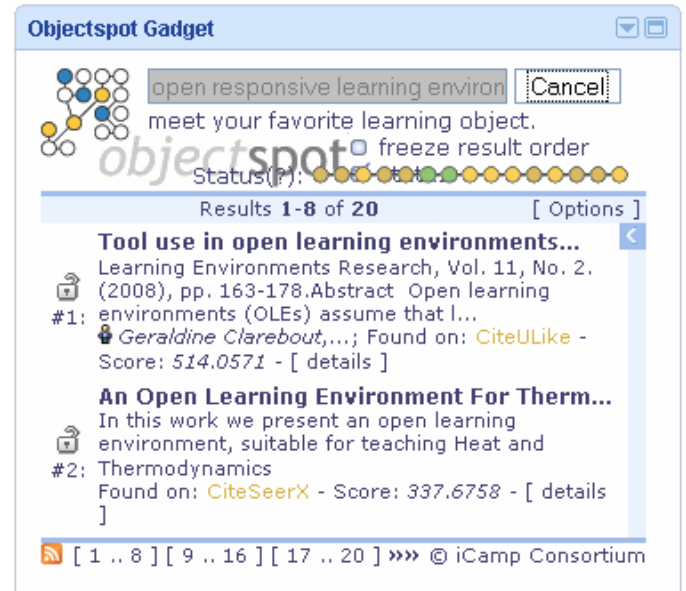

Figure 10. Objectspot - Federated Search Widget

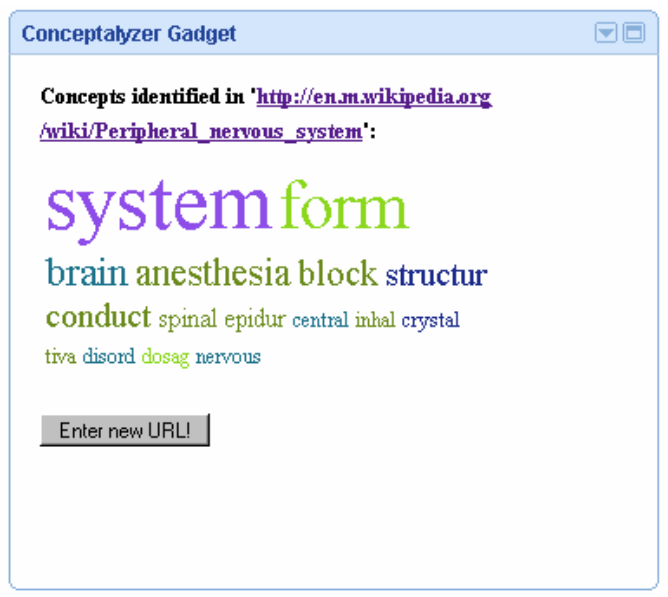

Figure 11. Conceptalyzer - Language Processing Widget

thus i s not forwar d com patible - an essent ial propert y, when w orking wi th experim ental sy stems. Given the diversity of devi ces and platforms available to the developers, we quickly had to find out that platform and bro wser independence was not given at all. Container-side or/and browser-side e rrors were the result. The most essential problem was the inaccessibility of bugs in JavaScript code. In many cases, problems occurred outside the source code under developer control. The reason was a malfunction in the code pr oduction performed by the cont ainer itself. Furthe rmore, error $m$ essages were cryptic and incomprehensible and thus did not provide any hint to the original location of an error. Furthermore, we lo st a lot of time communicating possible alternatives. An excursion to the usage of Apache Shindi g instead of SocialSite was also not successful for al 1 of us. Furt her problems were related to the incompatibility of external JavaScript libraries with the Widget co ntainer, wh ich ag ain resu lted in strange code $r$ ewriting effect $s$. Especi ally with regard $t o$ JavaScript 1 ibrary sup port for XM PP, we had to ex perience that libraries were not far enough for the realization of our goals and definitely need improvement. Finally, we had to expe rience that the d eveloper s upport by the S ocialSite team was not available at all. At the time of writing this document, it seems quite obvious, that SocialSite is dead.

We finally managed to deploy our prototype in Graaasp in a rather stable version, but still with a lot of open issues 
to $b$ e tack led in later $d$ evelopment s tages of the R OLE project.

Drawing the conclusions from our experiences, we can state $t$ hat $t$ he technologies we experi mented wi th were insufficiently mature for the deployment of a stab le integrated prototype assembled from a set of innovative tools realized using different technologies. For further collaborative distributed development experiments we agreed on short, but regular biweekly meetings in order to get aware of occurring problems earlier. The agenda will be inspired by action items of $\mathrm{W} 3 \mathrm{C}$ meetings.

\section{CONCLUSION \& OUTLOOK}

In th is paper we provided a rep ort of the collaborative distributed dev elopment of $t$ he R OLE C hristmas Project resulting in a prototype of a Widget-based PLE $f$ or language learning. We first described the development process cond ucted am ong ni ne different part ners from bot $h$ academia and industry, with varying tec hnical backgrounds, $\mathrm{m}$ otivations and $\mathrm{i}$ nterests regardi ng the $\mathrm{w}$ hole project. We pointed out th at regu lar co mmunication and the clear definition of goals and a schedule was inevitable during the w hole process. F urthermore, w e 1 isted useful technical means of c ollaboration suc $h$ as co mmunication media, shared docum entation, share d code repositories, etc. Furthermore, we had $\mathrm{t} o$ draw the conclusion that our approach did not work as expected, rising the necessity for an improved approach better suited for col laborative distributed development of W idget-based PLEs. In a section on requirements we presente $d$ our use case scenario a nd gave an i nsight i nto the psy cho-pedagogical model behind. In that cont ext we p ointed out that concept ual and technical work must happen together. We elicited technical requirem ents to a basic in frastructure for distributed PLE devel opment and presen ted a sel ection of $t$ echnologies for its realization as fo undation for our experiments. We then provided an overview of the inn ovations resulting from i ndividual part ner cont ributions, rangi ng fr om integration t echnologies to s cenario-dependent and i ndependent learn ing serv ice wid gets. Fin ally, we critica lly discussed the outcome of the ROLE Christmas project and reported a set of technical issues hinting to the conclusion, that Widget technology is not mature and stable enough to enable distributed collaborative PLE development without hassle at this point in time. However, we wo rked out the requirements and associ ated problems for di stributed implementation of wi dget based PLEs and col lected a lot of valuable experience that will shape future endeavors. In an upcoming con solidation pha se, the devel oper t eam wi ll stabilize cu rrent resu lts, i mprove an d alig $n$th e d evelopment process and then continue work towards a number of bundles for the implementation of the ROLE test bed scenarios.

\section{ACKNOWLEDGMENT}

The research leading to thes e results has received funding from the European Community's Seventh Framework Programme (FP7/ 2007-2013) un der grant agreement no 231396 (ROLE project).

\section{REFERENCES}

[1] K. Beck, M. Beedle, A. van Benneku m, A. Cockburn, W. Cunningham, M.Fowler et al. Manifesto for Agile Software Development. 2001. http://www.agilemanifesto.org/
[2] K. Beck and C. Andres. Extreme Programming Explained. Embrace Change. 2n d E dition, Addiso n W esley, Dece mber 2004, ISBN 0-321-27865-8

[3] git. T he fast ver sion contr ol sy stem. http://git-scm.com/. L ast visited: Jan 2010.

[4] A. Nussbaumer, K. Fruhmann, U. Kirschenmann, P. Ferdinand, A. Kiefel, A. Naeve . ROLE Deliver able 6.1: C ommon psychopedagogical framework. Unpublished.

[5] Apache Shindig. OpenSocial cont ainer reference implementation. http://incubator.apache.org/shindig/. Last visit January 2010.

[6] OpenSocial. A co mmon Web API f or social applications. http://code.google.com/apis/opensocial/. Last visit January 2010.

[7] Glassfish - Project SocialSite. https://socialsite.dev.java.net/. Last visit January 2010.

[8] Apache W ookie. http://incubator.apache.org/wookie/. Last visit January 2010.

[9] Marcos Cáceres . Widget Packaging and Configuration. W3 C Candidate Recommendation. W3C. December 2009.

[10] Open Ajax Al liance. Standa rdizing Ajax Develop ment. http://www.openajax.org. Last visit January 2010.

[11] Open Application. http://code.google.com/p/open-app/. Last visit January 2010.

[12] P. Saint-Andre, ed . Extensible Messaging and Presence Protocol (XMPP): Core, IE TF proposed standar d, RFC 3920, Oct. 2004; http://www.ietf.org/rfc/rfc3920.txt.

[13] P. Saint-Andre, ed . Extensible Messaging and Presence Protocol (XMPP): Instant Messaging and Presence, IE TF proposed standard, RFC 3921, 2004; http://www.ietf.org/rfc/rfc3921.txt

[14] P. Millard, P. Saint- Andre, Ralph Meijer, ed. XEP-060 PublishSubscribe. Dr aft Standar d in pr ogress, Dec. 2009; http://xmpp.org/extensions/xep-0060.html

[15] P. Saint-Andre, K. Smith, ed. XEP-163 Personal Eventing Protocol. Dr aft Standar d in pr ogress, Oct. 2009; http://xmpp.org/extensions/xep-0163.html

[16] I. Hickson and D. Hyatt. HTML5: A vocabulary and associated APIs for HTML and XHTML. E ditor's Draft. W3C. Jan. 2010; http://dev.w3.org/html5/spec/spec

[17] M. Wolpers, J. Najjar, K. Verbert, and E. Duval, ,Tracking Actual Usage: the Attenti on Me tadata Approach," in Educational Technology \& Society, vol. 10, no. 3, pp. 106-121, 2007.

[18] Apache Axis2: Ja va Next Gene ration W eb Ser vices. L ast updated: 23 Oct 2009; http://ws.apache.org/axis2

[19] Gears. I mproving Your W eb Br owser; http://gears.google.com/. Last visit January 2010.

[20] SQLite; http://www.sqlite.org. Last visit January 2010.

[21] U\&I Learning; http://www2.learning-service.com/portal/uni/. Last visit January 2010.

[22] A. I nselberg, "N- Dimensional Co ordinates," in IEEE Pattern Analysis \& Machine Intelligence (PAMI), "Picture Data Description \& Management", Asilomar, California, 1980, 136.

[23] S. Leitner: So lernt man lernen. 13. Auflage. Verlag Herder, Freiburg 1995, ISBN 3-451-05060-9

[24] B. Simon, D. Massart, F. van Assche, S. Ternier, and E . Duval. Simple Query Int erface Specificati on. Public Draft. April 2005; http://ariadne.cs.kuleuven.be/lomi/index.php/LorInteroperability

[25] R. Koblischke, Federated Ranking: Evaluating Result Merging Algorithms for Distributed Retrieval, master's thesis, Vienna University of Economics and Business, Vienna, 2010.

[26] F. Wild, B . Hoisl, and G. Bu rek, "Positioning for Conceptual Development using Latent Se mantic Analysis," in Proceedings of the EACL Workshop on GEMS, Athens, Greece, pp. 41-48, 2009.

\section{AUTHORS}

D. Renzel (re nzel@dbis.rwth-aachen.de) is with the Chair for Computer Science 5, RWTH Aachen University, Aachen, Germany.

C. Höbelt (christina.hoebelt@im-c.de) is with i mc in formation multimedia co mmunication $\mathrm{AG}$, Saarbr ücken, Germany. 
D. Dahrendorf ( $\mathrm{d}$ aniel.dahrendorf@im-c.de) is with imc i nformation m ultimedia co mmunication A G, Saarbrücken, Germany.

M. Friedrich ( martin.friedrich@fit.fraunhofer.de) is with th e Fraun hofer In stitute fo $\mathrm{r}$ Ap plied In formation Technology, Sankt Augustin, Germany.

F. Mödritscher (fm oedrit@wu.ac.at) is with th e Vienna University of Economics and Business, Vienna, Austria.

K. Verbert (katrien.verbert@cs.kuleuven.be) i s wi th the Katholieke Universiteit Leuven, Leuven, Belgium.
S. Govaerts (sten.govaerts@cs.kuleuven.be) is with the Katholieke Universiteit Leuven, Leuven, Belgium.

M. Palmér (matthias@nada.kth.se) is with the Uppsala University, Uppsala, Sweden.

Evgeny Bogdanov (evgeny.bogdanov@epfl.ch) is with the Écol e Pol ytechnique Fé dérale de La usanne (E PFL), Lausanne, Switzerland.

This article was modified from a presentation at the International Conference of Interactive Computer Aided Learning ICL2009, September 2009 in Villa ch, Aust ria. Sub mitted 11 Se ptember 2009. Published as resubmitted by the authors on 17 January 2010. 